\title{
Thyrotoxicosis leading to adrenal crises reveals primary bilateral adrenal lymphoma
}

\author{
Catarina Roque1, Ricardo Fonseca', Carlos Tavares Bello', Carlos Vasconcelos', \\ António Galzerano² and Sância Ramos² \\ ${ }^{1}$ Endocrinology Diabetes and Metabolism Clinic and 2Anatomopathology Department, Hospital de Egas \\ Moniz C.H.L.O.-E.P.E, Lisbon, Portugal
}

\author{
Correspondence \\ should be addressed \\ to C Roque \\ Email \\ barreiroscatarina@hotmail. \\ com
}

\section{Summary}

Primary adrenal lymphoma is a rare malignancy. It frequently presents bilaterally and with symptoms of adrenal insufficiency. Amiodarone may induce secondary organ dysfunction, and thyrotoxicosis develops in $15 \%$ of cases. The symptomatology of both conditions is nonspecific, especially in the elderly, and a high suspicion index is necessary for appropriate diagnosis. A 78-year-old female presented to the emergency department with confusion, nausea and vomiting. She had recently been to the emergency department with urinary tract infection, vomiting and acute hypochloremic hyponatremia. Upon re-evaluation, the leukocyturia persisted and because of TSH $0.01 \mu \mathrm{U} / \mathrm{mL}$ and free-T4 68 (10-18) pmol/L, she was admitted to the Endocrinology ward. Further evaluation supported amiodarone-induced thyroiditis type 2. Sepsis ensued, in the setting of nosocomial pneumonia. Hemodynamic instability, hyponatremia, hypoglycemia and vomiting raised the suspicion of adrenocortical insufficiency. Fluid resuscitation and hydrocortisone led to clinical improvement, and adrenal insufficiency was admitted. The thoracoabdominal tomography suggested an endobronchic primary lesion with hepatic and adrenal secondary deposits $(6.6$ and $7 \mathrm{~cm})$, but this was confirmed neither on pleural effusion nor on bronchofibroscopic fluid analyses. The adrenals were not accessible for biopsy. Despite high-dose hydrocortisone maintenance, the patient died before definite diagnosis. The autopsy confirmed primary non-Hodgkin lymphoma.

\section{Learning points:}

- Primary adrenal lymphoma is a rare cause of adrenal insufficiency, but progression can be fast and fatal.

- Hyperpigmentation is frequently absent.

- The presenting symptoms are nonspecific and might mimic infection. Disproportion of the general state with signs of specific organ symptomatology is a diagnostic clue.

- Infection may precipitate adrenal crisis and worsen thyroid function with further adrenal insufficiency exacerbation.

- In the context of thyrotoxicosis, there may be little clinical response to a therapeutic trial with standard dose glucocorticoids.

- High-dose glucocorticoid substitution may be required to achieve clinical stability in thyrotoxic patients.

\section{Background}

Primary adrenal lymphoma is a rare malignancy. Amiodarone-induced thyrotoxicosis develops in 15\% of amiodarone-treated patients. The symptomatology of both conditions is nonspecific, and a high suspicion index is necessary for appropriate diagnosis. Frailty, comorbidities and medications, may further delay timely diagnosis, especially in the elderly.

We report a case of adrenal crises and thyrotoxicosis with superimposed bacterial infection, of challenging diagnosis and management. We report the rare occurrence 
of primary adrenal lymphoma, revealed in the context of amiodarone-induced thyrotoxicosis. We stress the need of high-dose hydrocortisone maintenance in the setting of high free T4 levels.

\section{Case presentation}

A 78-year-old woman presented to the emergency department with confusion, nausea and vomiting on the 26th of April 2014. The complaints had started 4 weeks earlier. She was not able to report her own history, a family member did so.

She had type 2 diabetes mellitus, diagnosed in 1999, treated with metformin $1000 \mathrm{mg}$ q.d. and acarbose $50 \mathrm{mg}$ t.i.d. She also had history of hypertension controlled with $4 \mathrm{mg}$ of perindopril, paroxystic atrial fibrillation diagnosed in 2010 treated with amiodarone $200 \mathrm{mg}$ q.d. and warfarin, acute ischemic stroke in 2012, vascular dementia and a traumatic L1 fracture submitted to vertebroplasty in 2012. She was also medicated with simvastatin $20 \mathrm{mg}$ and trazodone $150 \mathrm{mg}$ q.d.

On the 18th of April, she had been observed by a medical team due to the same complaints. Longlasting dry cough and recent fasting hypoglycemia were also reported. By then, she was oriented and had a painful abdomen. The blood and urine tests showed hypochloremic hyponatremia, leukocytosis, leukocyturia and positive nitrites; therefore, she was diagnosed with a urinary tract infection (UTI) and discharged with antibiotic therapy and the recommendation to stop trazodone and acarbose and substitute the angiotensinconverting-enzyme inhibitor for an angiotensin-receptor antagonist (ARA).

One week later, medical observation was again sought. She had not complied with the recommendations. The patient was partially oriented, slightly dehydrated and the abdominal palpation elicited pain. The blood pressure and heart rate were normal, the rhythm was regular and the chest evaluation did not reveal any pathological findings. She had bilateral ankle edema. The blood-gas analysis was normal. Chest X-rays showed an enlarged heart and signs of interstitial pulmonary congestion. Pathological findings on blood tests: glucose: $66(70-100) \mathrm{mg} / \mathrm{dL}$, sodium: 125 (135-145) mmol/L, CRP: $7 \quad(<1) \mathrm{mg} / \mathrm{dL}$, TSH: $0.01(0.45-4.5) \mu \mathrm{U} / \mathrm{mL}$, free-T4: 68 (10-18) pmol/L, free-T3: 6.34 (4-8) pmol/L and leukocyturia.

The following conditions were postulated: persistent UTI, congestive heart failure, amiodarone-induced thyroiditis for which methimazole and prednisolone were started, hypotonic hypervolemic hyponatremia in the context of heart failure. At this point, she was transferred to the Endocrinology ward.

\section{Investigation and treatment}

During the first $24 \mathrm{~h}$, her clinical status worsened. There were noticeable pulmonary rhonchi and left-sided crackles, tachypnea and morning fever. The peripheral oxygen saturation and the blood pressure were low (systolic 90-100 and diastolic 52-59 $\mathrm{mmHg}$ ) but she was responding to fluid-challenge, with good diuresis. Empiric antibiotics were started after blood withdrawal for microbiology, and a bolus of hydrocortisone $100 \mathrm{mg}$ was given. The ARA was stopped, therapy was adjusted to methimazole $30 \mathrm{mg} /$ day and methylprednisolone $40 \mathrm{mg} /$ day e.v., and support measures were maintained. She was euglycemic without hypoglycemic agents, sodium was $129 \mathrm{mg} / \mathrm{dL}$, potassium: $3.8 \quad(3.5-4.5) \mathrm{mmol} / \mathrm{L}$, serum osmolarity: $281 \mathrm{mosmol} / \mathrm{L}$, uricemia: $3 \quad(<6.5) \mathrm{mg} / \mathrm{dL}$, urinary osmolarity: $566 \mathrm{mosmol} / \mathrm{L}$, natriuria: $133 \mathrm{mmol} / \mathrm{L}$, urine specific gravity: 1017 and NT-pro-BNP: $3780(<500)$ pg/mL. She had 10500 leukocytes/ $\mu \mathrm{L}$ with 90\% neutrophils, CRP: $30.2 \mathrm{mg} / \mathrm{dL}$, urea: $49(<17) \mathrm{mg} / \mathrm{dL}$ and creatinine: 0.96 $(<1.2) \mathrm{mg} / \mathrm{dL}$. The hypothesis of adrenal insufficiency (AI) was discussed but sepsis was the diagnosis most agreed with, plus, she was being administered glucocorticoids over the recommended stress dose. A short ACTH test was programmed to be performed as soon as the clinical situation would allow. In $48 \mathrm{~h}$, she was biochemically ameliorated, hemodynamically stable, apyretic, oriented and she had a functioning oral route. Cultures isolated Klebsiella pneumoniae and Pseudomonas aeruginosa, and antibiotics were adjusted by sensitivity tests.

The thyrotoxicosis investigation supported amiodarone-induced thyroiditis type 2. Thyroid ultrasound showed a hypoechoic heterogeneous parenchyma with decreased vascular signal on Doppler evaluation; anti-thyroglobulin, anti-thyroperoxidase and anti-TSH-receptor antibodies were negative.

Hypertension and hyperglycemia developed and required treatment for correction. An attempt to reduce the prednisolone dosage led to decreased level of consciousness, hypotension, hyponatremia and abdominal pain. There was no doubt that she had AI. Treatment was instituted as for adrenal crisis, and she was posteriorly maintained on oral hydrocortisone $150 \mathrm{mg}$ daily, in divided doses.

During the hospitalization, she acquired another pulmonary infection. It was homolateral to the previous and the X-ray showed left-sided pleural effusion and 
atelectasis of the inferior lobe. On blood tests, sodium was $132 \mathrm{mg} / \mathrm{dL}$, potassium $4.7 \mathrm{mg} / \mathrm{dL}$, magnesium 1.4 (2-4) $\mathrm{mg} / \mathrm{dL}$, free-T4 raised to $74 \mathrm{pmol} / \mathrm{L}$, free-T3 was $6.3 \mathrm{pmol} / \mathrm{L}, \mathrm{LDH} 1126(<280) \mathrm{U} / \mathrm{L}$ and the CRP went up to 20.1 from $2 \mathrm{mg} / \mathrm{dL}$ the previous day. Enterococcus spp was isolated.

At this point, we had the result of the abdominal ultrasound. There were bilateral adrenal solid masses, heterogeneous, with $66 \times 34 \mathrm{~mm}$ on the right and $70 \times 52 \mathrm{~mm}$ on the left side. On scanning the thorax and abdomen with contrasted tomography, some findings were clarified: there was complete atelectasis of the left inferior lobe, significant effusion and complete bronchial obliteration, findings suggesting an endobronchic lesion. The liver had 3 hypodense nodules, the larger with $14 \mathrm{~mm}$, which retained contrast at the periphery and could therefore represent secondary deposits. The adrenal lesions were heterogeneous, with areas of necrosis and enhanced heterogeneously, mainly at the periphery on the arterial phase. The left-sided mass was extending down to the renal hilum, compressing the renal pelvis, pressing part of the renal parenchyma, and it seemed to penetrate the renal vessels (Fig. 1). The bronchofibroscopy operator agreed with the suggestion of an endobronchic lesion. Analysis for mycobacteria, bacteria, fungi and malignant

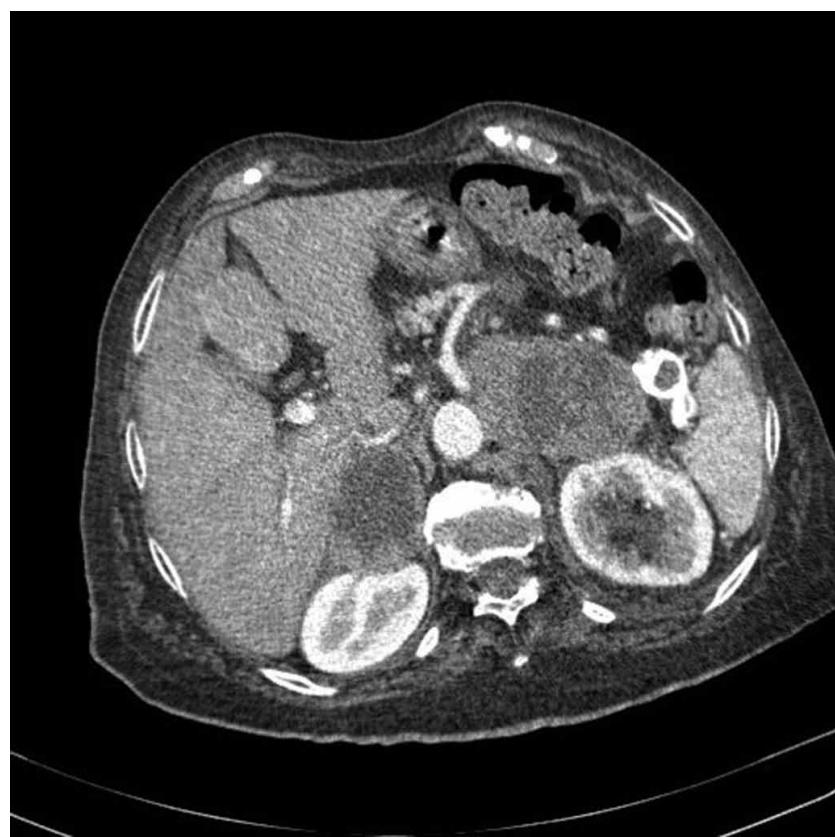

Figure 1

Abdominal contrasted computer tomography. Bilateral adrenal heterogeneous masses with areas of necrosis and late contrast retention. On the left side, there was ureteral compression and invasion of the kidney. cells were all negative. Thoracocentesis was then performed, the fluid being compatible with a transudate and negative on microbiological and cytological analysis. Revision of organs and past history did not suggest other possible primary origin of the tumor.

Blood test results for cortical and medullary adrenal function were awaited and biopsy was programmed, but none of the qualified radiologists agreed to perform adrenal nor liver biopsy, due to anatomical and technical difficulties that increased the risk of the procedure significantly.

The patient was stable, clinically and biochemically, but after this period of seemingly favorable evolution, the patient died.

\section{Outcome and follow-up}

Autopsy and histopathology confirmed primary bilateral adrenal non-Hodgkin lymphoma (PAL) of diffuse B cell type (Fig. 2).

On the right side, the mass had $95 \mathrm{~mm}$ and a thin rim of adrenal tissue was identified but on the left side, it had $135 \mathrm{~mm}$ and no adrenal tissue could be found.

The following results were obtained after the patient's death: 24-h urinary metanephrines $27.3 \quad(<350) \mu \mathrm{g} /$ $\mathrm{mL}, 24$-h urinary normetanephrines $136(<139) \mu \mathrm{g} / \mathrm{mL}$, DHEA-sulfate $<20(35-430) \mu \mathrm{g} / \mathrm{dL}$, total testosterone $<20$ $(<43) \mathrm{ng} / \mathrm{dL}$, renin concentration $7.3 \mathrm{ng} / \mathrm{L}$, aldosterone

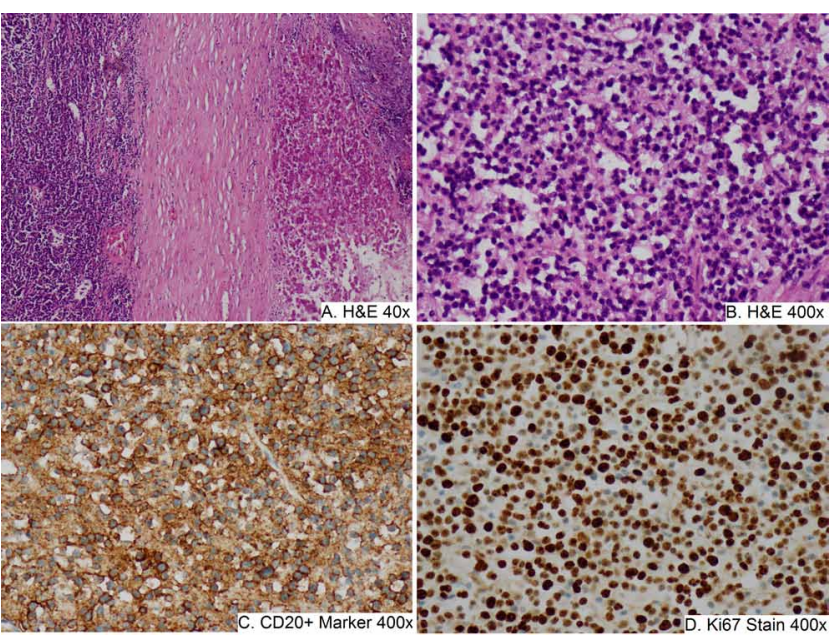

Figure 2

(A) Diffuse neoplasia is observed on the left, separated by a layer of fibrosis from the remnant adrenal tissue on the right side of the image. (B) Medium-to-large atypical lymphoid cells and areas of spontaneous necrosis. (C) CD 20 positivity identifying B cell phenotype. (D) High proliferative index. 
$<1.1$ (1-16) pg/mL, ACTH <5 (<46) pg/mL, CEA $13.5(<5)$, CA125 $425(<35)$; CA19.9, CA15.3 and NSE were normal.

\section{Discussion}

The adrenal gland is an extremely rare site of primary extranodal non-Hodgkin lymphoma occurrence $(<1 \%$ of cases). It usually presents bilaterally and with AI $(1,2)$.

Regardless of its rarity, it must always integrate the differential diagnosis, especially in the elderly patient, where its incidence is greater, symptoms are subtler and progression is often fast and fatal (3).

Due to the lack of pathognomonic symptoms or signs, early diagnosis may be difficult (3). Fever of unknown etiology and infection have been reported as the most commonly co-occurring diagnosis in patients presenting with adrenal crises (4) and have also been described frequently in the reports of PAL. Fever has been found to be, together with pain, the most commonly presenting sign (5).

The most specific sign of primary AI is hyperpigmentation, but in this case, it was absent. It has been reported to occur in a minority of patients diagnosed with PAL, which might reflect the aggressive nature of this specific pathology (6). We revised the tomography of 2012, directed to the lumbar pathology and no adrenal lesions were seen.

The radiologic findings of PAL are not specific, and the differential diagnosis is broad. Bilateral adrenomegaly, in the setting of acquired hypocortisolism, most likely represents an infiltrative process of infectious, neoplastic, hemorrhagic, granulomatous or deposit (hemochromatosis and amyloidosis) etiology. Infectious lesions (tuberculosis and less often histoplasmosis, blastomycosis, CMV and candidiasis) usually present with signs of involvement elsewhere in the body. Neoplasms may be primary (adenomas, pheochromocytomas and lymphoma) or metastatic (lung, breast, colon, kidney, melanoma and lymphoma) and commonly present with a known primary lesion. In the elderly population, tuberculosis and malignant disease are the most common causes. Radiologic findings may be suggestive but are not diagnostic. PAL reports usually show large well-defined infiltrative masses of variable density and enhancement patterns (7). Central necrosis is not typical, but it was present in our case. The most distinguishing feature is perhaps minimal displacement of the surrounding structures despite its size and infiltrative behavior (8).
In the present case report, infection was present ad initium, confirmed microbiologically, and it surely worsened the thyrotoxicosis and the adrenal insufficiency. Looking retrospectively, if infection had not been documented, the diagnosis could have been both adrenal crises and thyroid storm, according to the Akamizu's diagnostic criteria. One should note although, that infection is commonly coexistent, so its confirmation should not redirect the physician away from the crisis hypothesis.

Thyrotoxicosis, especially when due to amiodaroneinduced destruction, may be difficult to control rapidly. This thyrotoxic state leads to increased substitutive hydrocortisone requirements (9) that in our case were as high as $150 \mathrm{mg} /$ day.

Transient and unpredictable periods of worsening thyroid function occur and associate with infectious processes. These fluctuations in free-T4 levels may require glucocorticoid dose adjustments and are of difficult management in the short term. Mortality rises significantly.

\section{Declaration of interest}

The authors declare that there is no conflict of interest that could be perceived as prejudicing the impartiality of the research reported.

\section{Funding}

The reported work did not receive any specific grant from any funding agency in the public, commercial or not-for-profit sector.

\section{Patient consent}

Informed consent for imaging publication has been obtained from a relative. Unfortunately, the patient reported in the case has passed away.

\section{Author contribution statement}

Catarina Roque - primary physician of the patient. Catarina Roque, Ricardo Fonseca, Carlos Tavares Bello, Carlos Vasconcelos - the medical team that assisted the patient. António Galzerano and Sância Ramos - the anatomopathologists who performed the autopsy and histological studies.

\section{References}

1 Singh D, Kumar L, Sharma A, Vijayaraghavan M, Thulkar S \& Tandon N 2004 Adrenal involvement in non-Hodgkin's lymphoma: four cases and review of literature. Leukemia and Lymphoma 45 789-794. (doi:10.1080/10428190310001615756)

2 Rashidi A \& Fisher SI 2013 Primary adrenal lymphoma: a systematic review. Annals of Hematology 92 1583-1593. (doi:10.1007/s00277-0131812-3) 
3 Kung AW, Pun KK, Lam K, Wang C \& Leung CY 1990 Addisonian crisis as presenting feature in malignancies. Cancer 65 177-179. (doi:10.1002/1097-0142(19900101)65:1<177::aidcncr2820650134>3.0.co;2-8)

4 Rushworth RL \& Torpy DJ 2014 A descriptive study of adrenal crises in adults with adrenal insufficiency: increased risk with age and in those with bacterial infections. BMC Endocrine Disorders 1479. (doi:10.1186/1472-6823-14-79)

5 Mozos A, Ye H, Chuang WY, Chu JS, Huang WT, Chen HK, Hsu YH, Bacon CM, Du MQ, Campo E, et al. 2009 Most primary adrenal lymphomas are diffuse large B-cell lymphomas with nongerminal center B-cell phenotype, BCL6 gene rearrangement and poor prognosis. Modern Pathology 22 1210-1217. (doi:10.1038/ modpathol.2009.87)
6 Wang J, Sun NCJ, Renslo R, Chuang CC, Tabbarah HJ, Barajas L \& French SW 1998 Clinically silent primary adrenal lymphoma: a case report and review of the literature. American Journal of Hematology 58 130-136. (doi:10.1002/(sici)1096-8652(199806)58:2<130::aid-ajh8>3.3.co;2-0)

7 Zhou L, Peng W, Wang C, Liu X, Shen Y \& Zhou K 2012 Primary adrenal lymphoma: radiological; pathological, clinical correlation. European Journal of Radiology 81 401-405. (doi:10.1016/j. ejrad.2010.11.026)

8 Johnson P, Horton KM \& Fishman EK 2009 Adrenal mass imaging with multidetector CT: pathologic conditions, pearls and pitfalls. RadioGraphics 29 1333-1351. (doi:10.1148/rg.295095027)

9 Newrick PG 1984 Addison's disease and thyrotoxicosis presenting simultaneously. Postgraduate Medical Journal 60 478-479. (doi:10.1136/pgmj.60.705.478)

Received in final form 26 March 2017 Accepted 6 April 2017 